\title{
PREDICTIVE MODEL FOR NORMALIZED SHEAR MODULUS OF COHESIVE SOILS
}

\author{
Yaser JAFARIAN $^{1)}$ *, Abdolhosein HADDAD ${ }^{2)}$ and Hamed JAVDANIAN ${ }^{3)}$ \\ 1) Geotechnical Engineering Research Center, International Institute of Earthquake Engineering and Seismology, \\ Tehran,Iran,yjafarianm@iiees.ac.ir \\ 2) Department of Civil Engineering, Semnan University, Semnan, Iran, ahadad@semnan.ac.ir \\ 3) Department of Civil Engineering, Semnan University, Semnan,Iran, h.javdanian@semnan.ac.ir \\ *Corresponding author's e-mail: yjafarianm@iiiees.ac.ir
}

\begin{tabular}{|c|c|}
\hline ARTICLE INFO & ABSTRACT \\
\hline Article history: & \multirow{4}{*}{$\begin{array}{l}\text { Evaluating dynamic properties of geomaterials is an essential step for solving geotechnical } \\
\text { earthquake engineering problems. The shear stiffness-reduction curves of soils are commonly } \\
\text { presented in normalized form and have many applications in equivalent-linear and nonlinear } \\
\text { dynamic analyses. In this study, a radial basis function (RBF) neural network model was } \\
\text { developed to predict normalized shear modulus of cohesive soils. The most important factors } \\
\text { that affect this parameter include effective confining pressure, shear strain, and plasticity index. } \\
\text { The comprehensive database used for the development of the model was obtained from } \\
\text { previously published experimental results. Validation of the model performance was carried out } \\
\text { by using centrifuge tests results. A parametric analysis was then performed to evaluate } \\
\text { sensitivity of the proposed model to variations of the influencing parameters. The results } \\
\text { indicate that the neural network model could provide predictions more accurate than those } \\
\text { obtained by the previous models. }\end{array}$} \\
\hline $\begin{array}{l}\text { Received } 17 \text { December } 2012 \\
\text { Accepted } 9 \text { May } 2013 \\
\text { Available online } 26 \text { November } 2013\end{array}$ & \\
\hline Keywords: & \\
\hline $\begin{array}{l}\text { Dynamic properties } \\
\text { Shear modulus } \\
\text { Cohesive soils } \\
\text { Neural Network }\end{array}$ & \\
\hline
\end{tabular}

\section{INTRODUCTION}

Reliable and accurate curves of soils dynamic stiffness are necessary for the solution of many soil dynamic problems like vibration of machine foundations, response of soil deposits and earth structures to earthquake, evaluation of traffic vibration, and so forth. Various techniques might be used to solve the aforementioned problems. However, regardless of the procedure chosen, it is first necessary to evaluate the appropriate dynamic properties of the materials in the site. For example, to perform site response analysis, the normalized shear modulus curves of soil should be primarily evaluated. However, the range of shear modulus reduction and damping curves of soils are quite wide; hence, selections of dynamic curves have an important effect on the results of dynamic analysis. The range of variations in the selection of curves demonstrates the uncertainties associated with the nonlinear dynamic behavior of soils. Therefore, in these analyses, uncertainties must be reduced by predicting the shear modulus reduction curves with the most accurate methods.

The secant shear modulus, G, represents the shear stiffness of the soil in the given level of shear strain. It is essentially the slope of the shear stress $(\tau)$ and strain $(\gamma)$ curve. Because of the nonlinear nature of the stress-strain curve of soils, shear modulus of soils changes with strain amplitude. In the range of very small strains, soil behavior is approximately linear-elastic. The shear modulus at small strains is called maximum shear modulus, $G_{\max }$. It can be determined by in-situ methods from the measurements of the shear wave velocity, $\mathrm{V}_{\mathrm{s}}$, by means of crosshole, down-hole, up-hole, or surface wave testing. The relationship between maximum shear modulus and shear wave velocity is rigorous: $G_{\max }=\rho \cdot V_{s}^{2}$ where $\rho$ is soil density. This parameter can be also obtained in laboratory by resonant column or bender element tests.

Estimation of the dynamic properties (shear modulus, $G$, and damping ratio, $D$, of soils has become the scope of many studies in the field of geotechnical earthquake engineering using devices such as resonant column, cyclic simple shear, cyclic torsional shear and cyclic triaxial (e.g., Hardin and Drnevich, 1972a; Tatsuoka et al., 1979; Yoshimi et al., 1984; Wilson, 1988; Saxena and Reddy, 1989; Yasuda and Matsumoto, 1993; Konno et al., 1994; Lanzo et al., 1997; Menq, 2003; Hardin and Kalinski, 2005; Xenaki and Athanasopoulos, 2008; Senetakis et al., 2012a; Senetakis et al., 2012b).

Many studies have been conducted to characterize the factors that affect normalized shear modulus $\left(\mathrm{G} / \mathrm{G}_{\max }\right)$ and damping ratio (D) of soils (e.g., Richart et al., 1970; Hardin and Drnevich, 1972b; Vucetic and Dobry, 1991; Ishibashi and Zhang, 1993; Darendeli, 2001; Stokoe et al., 1994, 2004). Based on these studies, the most important factors that affect $\mathrm{G} / \mathrm{G}_{\max }$ of cohesive soils include shear strain $(\gamma)$, effective confining pressure $\left(\sigma^{\prime}\right)$, and plasticity index (PI). Other parameters have less important effect. 
Table 1 Descriptive statistics of the variables used in the model development.

\begin{tabular}{|c|c|c|c|c|c|}
\hline \multirow{2}{*}{ Statistical parameters } & \multirow[t]{2}{*}{ Subset } & \multicolumn{2}{|c|}{ Parameters } & \multirow[b]{2}{*}{$\gamma(\%)$} & \multirow[b]{2}{*}{$\mathrm{G} / \mathrm{G}_{\max }$} \\
\hline & & PI (\%) & $\sigma_{0}^{\prime}(\mathrm{kPa})$ & & \\
\hline \multirow{2}{*}{ Minimum } & Training & 5 & 16 & 0.0001 & 0.055 \\
\hline & Testing & 5 & 16 & 0.0001 & 0.059 \\
\hline \multirow[t]{2}{*}{ Maximum } & Training & 132 & 575 & 6 & 1 \\
\hline & Testing & 132 & 575 & 4.7 & 1 \\
\hline \multirow[t]{2}{*}{ S.D. ${ }^{a}$} & Training & 32.7 & 128.73 & 0.623 & 0.23 \\
\hline & Testing & 31.6 & 132.01 & 0.601 & 0.24 \\
\hline \multirow[t]{2}{*}{ Mean } & Training & 46 & 118.9 & 0.160 & 0.812 \\
\hline & Testing & 47.7 & 116.81 & 0.180 & 0.810 \\
\hline
\end{tabular}

${ }^{\mathrm{a}}$ Standard deviation

Zhang et al. (2005) presented predictive equations for estimating normalized shear modulus and damping ratio. Their equations are based on a modified hyperbolic model, which include some variables: shear strain amplitude, confining pressure, and plasticity index (PI). In their study, the database was categorized into three groups based on geological age: Quaternary soil, Tertiary and older soil, and residual/saprolite soil.

In the recent years, new aspects of modeling, optimization, and problem solving have been developed in light of the pervasive promotion in computational software and hardware. Some aspects of software engineering, referred to as artificial intelligence such as artificial neural networks (ANNs), are relied upon the iterative learning of databases. In case of complicated problems, experimentalists prefer these trial approaches rather than analytical optimization. Numerous researchers applied artificial intelligence approaches in the various fields of geotechnical engineering such as stress-strain modeling of soil (Penumadu and Zhao, 1999), slope stability (McCombie and Wilkinson, 2002), shallow foundations (Shahin et al., 2002), soil liquefaction (Baziar and Jafarian, 2007, Baziar et al., 2011; Ghorbani et al., 2012; Jafarian et al., 2013), and earthquake engineering (Jafarian et al., 2010).

As discussed above, numerous data of dynamic soil properties were measured in laboratory during the previous studies. The current study aims to propose a RBF-based neural network model for predicting the $\mathrm{G} / \mathrm{G}_{\max }$ of cohesive soils in terms of shear strain, $\gamma(\%)$, confining pressure, $\sigma_{0}{ }_{0}(\mathrm{kPa})$, and Plasticity index, PI (\%). The training process is carried out on a wide-ranging database of laboratory tests conducted during the previous studies. In order to ensure that the RBF model is sufficiently generalized, a seperate dataset including the results of centrifuge tests is used in the modeling. Parametric studies are carried out to demonstrate reasonable behavior of the proposed model. Outputs of the model are compared with the ranges proposed by the previous studies.

\section{DATA COLLECTION}

A wide-ranging database was compiled in the current study from the previously published laboratory tests (U.S. Army Corps of Engineers, 1978, 1979; Kokusho et al., 1982; Borden et al., 1994, 1996; Lanzo et al., 1997; S\&ME Inc., 1993, 1998; Stokoe et al., 1995; Caserta et al., 2012). These studies include numerous tests conducted on cohesive soils to measure their dynamic properties at low and large strain levels using resonant column, simple shear, cyclic torsional shear, and cyclic triaxial tests.

The database was divided into two separate groups denoted as training and testing sets consisting $80 \%$ and $20 \%$ of data, respectively. The testing set was utilized to determine when training should be stopped to avoid overfitting. The data division process was performed so that the main statistical parameters of the training and testing subsets (i.e., maximum, minimum, mean, and standard deviation) become close to each other. For this purpose, a trial selection procedure was carried out and the most possible consistent division was determined (Masters, 1993). The descriptive statistical parameters of these two groups are presented in Table 1.

The results of centrifuge tests (Brennan et al., 2005), as validation set, were also employed in addition to the training and testing sets for further examination and generalization of the model performance. The number of data considered for training, testing, and validation set is 544,136 , and 15 , respectively.

\section{ARTIFICIAL NEURAL NETWORK}

Artificial Neural network (ANN) is a functional abstraction of the biological neural structures of the central nervous system (Wasserman, 1993; Alexhander and Morton, 1993; Anderson 1995; Arbib, 1995). It can exhibit a surprising number of human brain's characteristics e.g. learn from experience and generalize from previous examples to new problems. These systems are capable of high-level functions, such as adaptation or learning, and lower level 


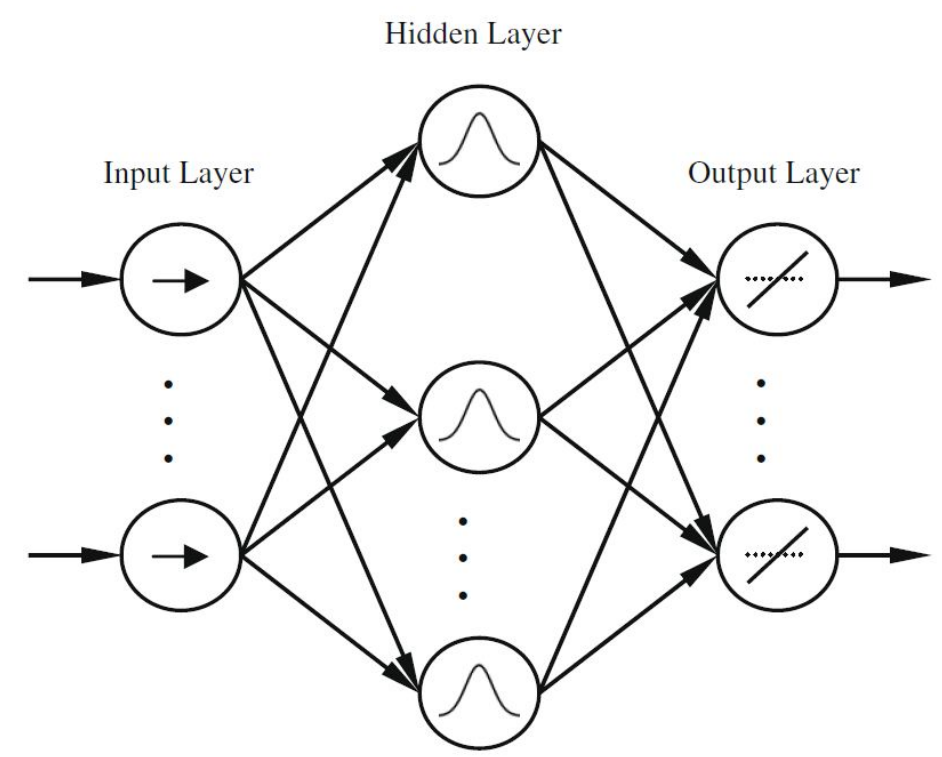

Fig. 1 Typical topology of RBF networks.

functions such as data pre-processing for different kinds of inputs. Neurons are the main processing elements of the ANNs. Details of the structure and operation of ANNs can be found in many publications (Smith, 1993; Fausett, 1994; Galushkin, 2007).

Feed forward back propagation algorithm could be mentioned as a sub-set of multi-layer perceptron neural networks. The topology of FFBP ANNs consists of a set of neurons connected by links in a number of layers. The basic configuration usually consists of an input layer, a hidden layer and an output layer. The number of hidden layers establishes the complexity of the network. Designating the correct number of hidden layers and the number of nodes in each layer has been evaluated by try and error. However, each node multiplies every input by its interconnection weights and then adds the sum of the products to a bias number, and then passes the sum through a transfer function to generate the results. The transfer function which is usually a sigmoid function can be presented as the Eq. 1 .

$y_{i}=f\left(\sum w_{i j} x_{i}+b_{i}\right)=\frac{1}{1+e^{-\left(\sum w_{i j} x_{i}+b_{i}\right)}}$

where $\mathrm{w}_{\mathrm{ij}}$ is the weight of the connection joining the $j$ th neuron in a layer to the $i$ th neuron in the previous layer and $\mathrm{x}_{\mathrm{i}}$ is the value of $i$ th neuron in the previous layer. Under the aegis of this function, the output $y_{j}$ from the $j$ th neuron in a layer could be calculated. After comparing the obtained results with the target values, the errors would be calculated and by using the back propagation algorithm the entire weights would be corrected. This process will be continued until either errors are less than a specified value or the number of training epochs reaches the favorite repetition. At this stage, ANN is considered as trained (Hornik et al., 1989).

\section{RBF NEURAL NETWORK}

Radial basis functions, which were first used by Broomhead and Lowe (1988), are powerful and interesting networks due to their rapid training, generality and simplicity. Girosi et al. (1991) and Hartman et al. (1990) proved that RBF networks are universal approximators and can approximate any continuous function with arbitrary accuracy.

The RBF neural network has three layers: the input, the hidden, and the output layer. The hidden layer involves RBF neurons with Gaussian activation functions. The outputs of RBF neurons have significant responses to the inputs only over a range of values of $X$ called the receptive field. The size of the receptive field is determined by the value of $\sigma$. Activation function of RBF neurons is as Eq. 2.

$$
\varphi_{i}=\exp \left(-\left(X-C_{i}\right)^{T}\left(X-C_{i}\right) /\left(2 \sigma_{i}^{2}\right)\right)
$$

where $X$ is an input vector, $\varphi_{i}, C_{i}$ and $\sigma_{i}$ are Gaussian activation function, weight vector, and radius of receptive field of $i$ th RBF neuron, respectively.

The value of $\sigma$ allows the sensitivity of the RBF neurons to be adjusted. During the training, the $\sigma$ value of RBF neurons is such determined as the neurons could cover the input space properly. The output layer neurons produce the linear weighted summation of hidden layer neurons responses according to Eq. 3 .

$y_{k}=\sum_{i=1}^{n} \varphi_{i} w_{k i}$

where $y_{k}$ is the $k$ th output and $w_{k i}$ is the weight from the $i$ th RBF neuron to the $k$ th output neuron and $\mathrm{n}$ is the number of RBF neurons.

The numerical results of many scientific and engineering applications indicate that RBF networks 
Table 2 Target statistical parameters to comparison of FFBP and RBF models.

\begin{tabular}{lllcccc}
\hline Group & \multicolumn{3}{l}{ Performance } \\
& \multicolumn{3}{l}{ FFBP model } & \multicolumn{3}{c}{ RBF model } \\
\cline { 2 - 8 } & $\mathrm{R}^{2}$ & MAE & RMSE & $\mathrm{R}^{2}$ & MAE & RMSE \\
\hline Training & 0.958 & 0.031 & 0.043 & 0.969 & 0.028 & 0.039 \\
Testing & 0.963 & 0.030 & 0.046 & 0.960 & 0.032 & 0.049 \\
All element tests & 0.961 & 0.033 & 0.044 & 0.967 & 0.028 & 0.042 \\
Validation (centrifuge) & 0.918 & 0.042 & 0.047 & 0.923 & 0.041 & 0.044 \\
\hline
\end{tabular}

are very good tools for interpolation and also their training is very fast. Typical topology of RBF networks is shown in Figure 1.

In this study, RBF neural network was used for the prediction of normalized shear modulus of cohesive soils. This network has 3 neurons in input layer, 3 neurons in hidden layer, and 1 neuron in output layer. Input parameters include: shear strain, effective confining pressure, and plasticity index of soil and output parameter is normalized shear modulus. In order to obtain a more efficient training process, the input and target were standardized to have zero mean and unity standard deviation. In this study, results of geotechnical centrifuge tests with 15 data pairs were used as validation set for further controlling on the training stoppage and increasing the generality of the model for future predictions.

In order to examine the robustness of the proposed models, the coefficient of determination $\left(\mathrm{R}^{2}\right)$, mean absolute error (MAE), and root mean squared error (RMSE) between the measured and predicted $G / G_{\max }$ ratios were obtained according to Eqs. 4-6.

$$
\begin{aligned}
& R^{2}=\sum_{N}\left(X_{m}\right)^{2}-\frac{\sum_{N}\left(X_{m}-X_{p}\right)^{2}}{\sum_{N}\left(X_{m}\right)^{2}} \\
& M A E=\frac{\sum_{N}\left|\left(X_{m}-X_{p}\right)\right|}{N}
\end{aligned}
$$

$R M S E=\sqrt{\frac{\sum_{N}\left(X_{m}-X_{p}\right)^{2}}{N}}$

where $N$ is the number of data, $X_{m}$ and $X_{p}$ are measured and predicted values, respectively.

Among the developed models, the RBF neural network model with the most great performance (greater coefficient of determination and smaller MAE and RMSE) for the training, testing, and validation data sets was selected. Since, the models were generated based on the element tests data and validated by the centrifuge tests, it is reasonable to anticipate that the models are generalized enough to predict real normalized shear modulus of cohesive soil.

\section{RESULTS}

Numerous runs were performed with various initial settings and the performance of the developed model was analyzed for each run. Consequently, the best model was selected according to the treated statistical criteria i.e. $\mathrm{R}^{2}, \mathrm{MAE}$ and RMSE. In addition, a comprehensive parametric study was performed to monitor the behavior of each model versus variations of input variables. The proposed predictive model involves three input parameters $(\gamma$, $\sigma^{\prime}{ }_{0}$, and $\left.\mathrm{PI}\right)$ and one output $\left(\mathrm{G} / \mathrm{G}_{\max }\right)$.

Two ANN models, feed forward back propagation, FFBP, and radial basis function, RBF, were utilized to predict the normalized shear modulus of cohesive soils. The results indicate that RBF model is a better model than the FFBP model in light of its simplicity and reasonable performance in shear modulus prediction. This is reflected by presenting the values of target statistical parameters in Table 2 . Therefore, in this study the detailed results of RBF model are presented.

Precision of the proposed model is examined by plotting the measured versus predicted values of $\mathrm{G} / \mathrm{G}_{\max }$ for training, testing, and all element tests data, as shown in Figures 2-4, respectively. The values of $\mathrm{R}^{2}$, MAE, and RMSE are respectively equal to 0.969 , 0.028 , and 0.039 for training sets (Fig. 2) and 0.960, 0.032, and 0.049 for testing sets (Fig. 3). For all data sets (Fig. 4), $\mathrm{R}^{2}$ is equal to 0.967 while MAE and RMSE are equal to 0.028 and 0.042 , respectively.

It was previously indicated that the centrifuge data set was included in the analysis to confirm enough generalization of the model for future predictions. The centrifuge tests data were adopted from the study conducted by Brennan et al. (2005) on clayey soil. In their studies, the centrifuge tests were carried out by Cambridge centrifuge, with $10 \mathrm{~m}$ diameter beam, under the centrifugal acceleration of $50 \mathrm{~g}$. The earthquake excitation was applied using the mechanical stored angular momentum actuator. 


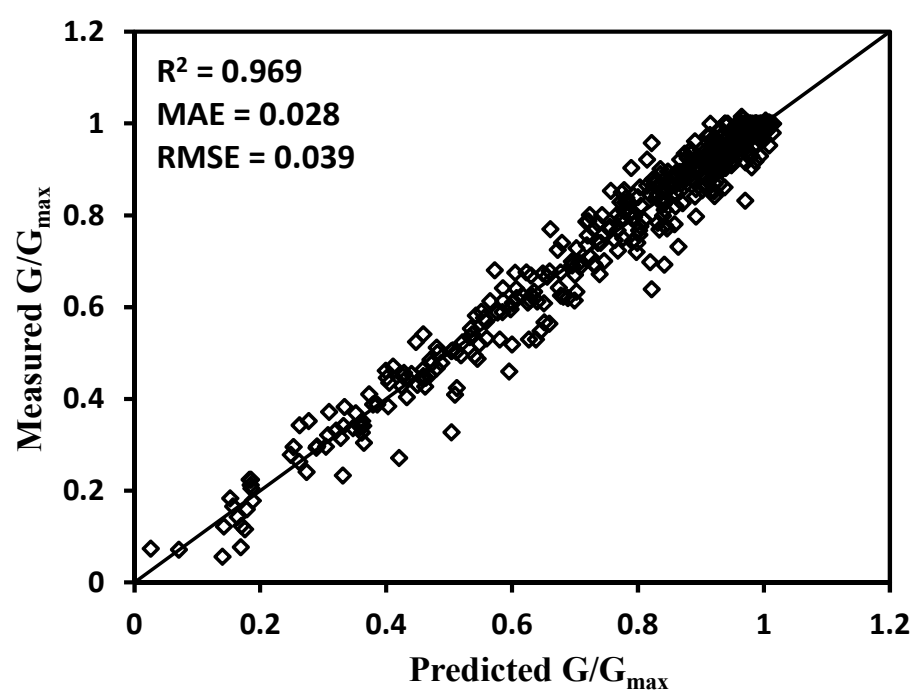

Fig. 2 Measured versus predicted values of $\mathrm{G} / \mathrm{G}_{\max }$ for training data set.

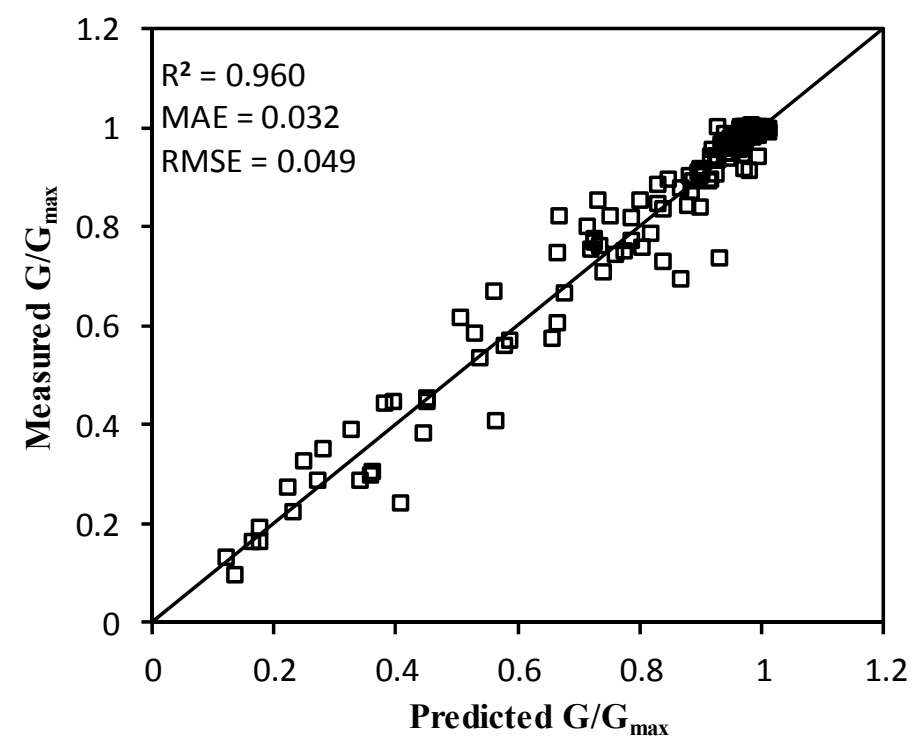

Fig. 3 Measured versus predicted values of $\mathrm{G} / \mathrm{G}_{\max }$ for testing data set.

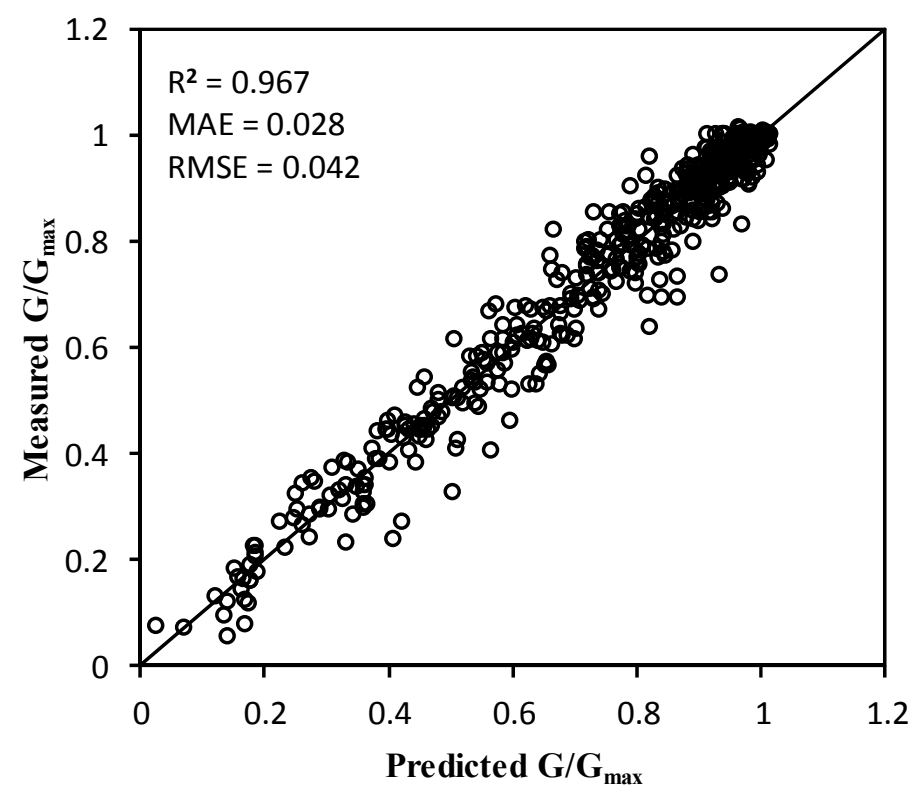

Fig. 4 Measured versus predicted values of $G / G_{\max }$ for all element tests data. 


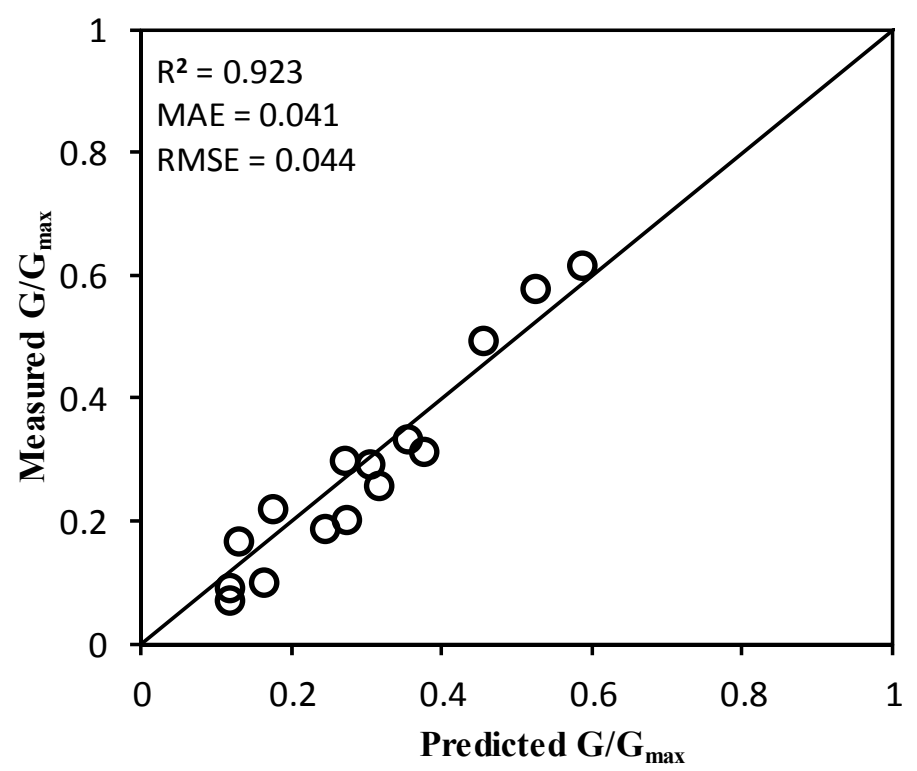

Fig. 5 Measured versus predicted values of $\mathrm{G} / \mathrm{G}_{\max }$ for centrifuge validation set.

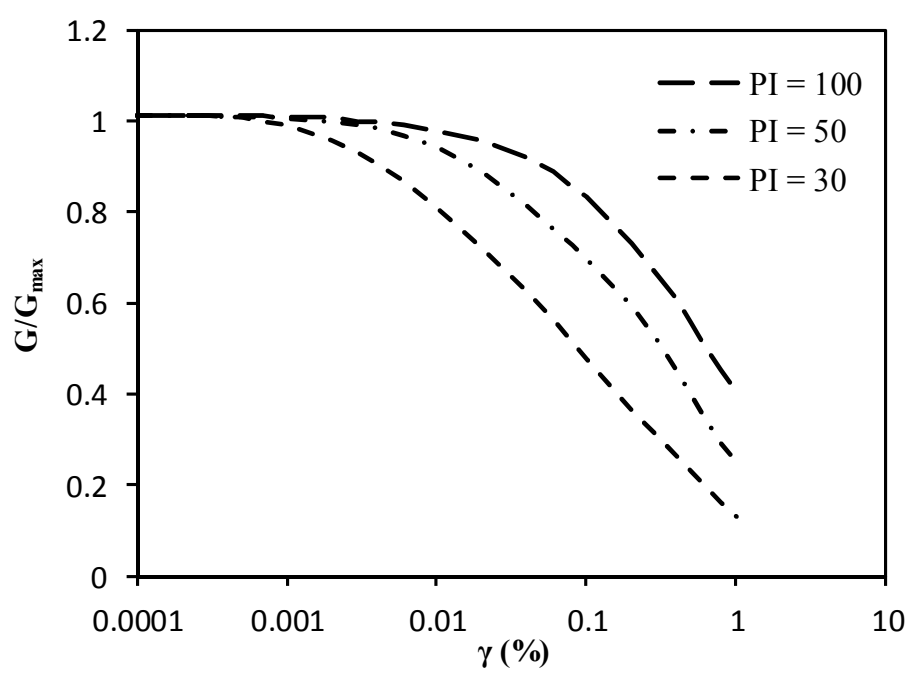

Fig. 6 Variation of $\mathrm{G} / \mathrm{G}_{\max }$ predicted by RBF-based model against $\gamma$ at different levels of PI.

Readers can refer to the original reference of this study (Brennan et al., 2005) for more details on the tests procedure and the processed $\mathrm{G} / \mathrm{G}_{\max }$ data. Figure 5 depicts measured versus predicted values of $\mathrm{G} / \mathrm{G}_{\max }$ for the validation data set. The values of $\mathrm{R}^{2}$, MAE, and RMSE for this data set were obtained equal to $0.923,0.041$, and 0.044 , respectively. In fact, the evolved model has obtained high accuracy for both testing and validation sets. From the plots shown in Figures 2-5, it is observed that the developed model can predict the $\mathrm{G} / \mathrm{G}_{\max }$ of cohesive soils with reasonable accuracy.

\section{PARAMETRIC ANALYSIS AND MODEL ACCURACY}

Further examination on the model performance under various conditions was conducted through a parametric analysis. This part of the study was performed to evaluate whether the model outputs are in accordance with the mechanisms found in the experimental investigations. For this purpose, variations of any input parameter on the amount of $\mathrm{G} / \mathrm{G}_{\max }$ were studied while the other input parameters were kept constant at their mean values in the data set.

Variations of the $\mathrm{G} / \mathrm{G}_{\max }$ data predicted by RBFbased model against $\gamma$ at different levels of PI are shown in Figure 6. The variations are similar to the results presented by Vucetic and Dobry (1991) for cohesive soils, because they showed that clays with more plasticity obtain larger $\mathrm{G} / \mathrm{G}_{\max }$ values at a certain level of shear strain amplitude. They concluded that plasticity index (PI) is the main factor controlling the modulus reduction curve $\left(\mathrm{G} / \mathrm{G}_{\max }-\gamma\right)$. Also, they concluded that soils with higher plasticity tend to have a more linear cyclic response at small strains and to degrade less at larger $\gamma$ than soils with a lower PI. Based on Vucetic and Dobry (1991)'s results $\mathrm{G} / \mathrm{G}_{\max }-\gamma$ curve moves up as the soil plasticity (PI) increases. 


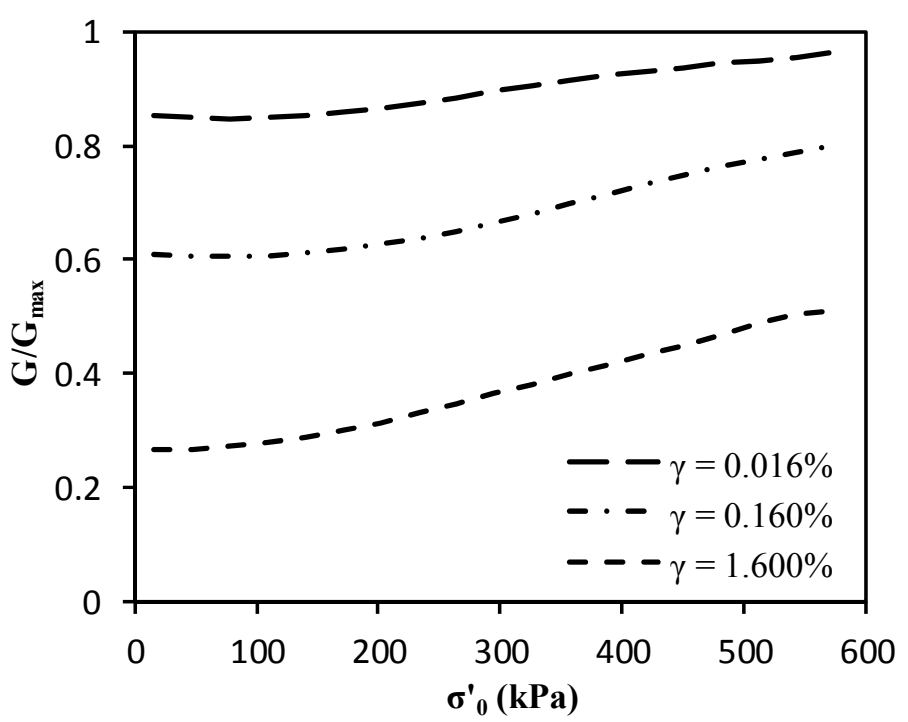

Fig. 7 Variation of $\mathrm{G} / \mathrm{G}_{\max }$ predicted by $\mathrm{RBF}$-based model against $\sigma_{0}^{\prime}$ at different levels of $\gamma$.

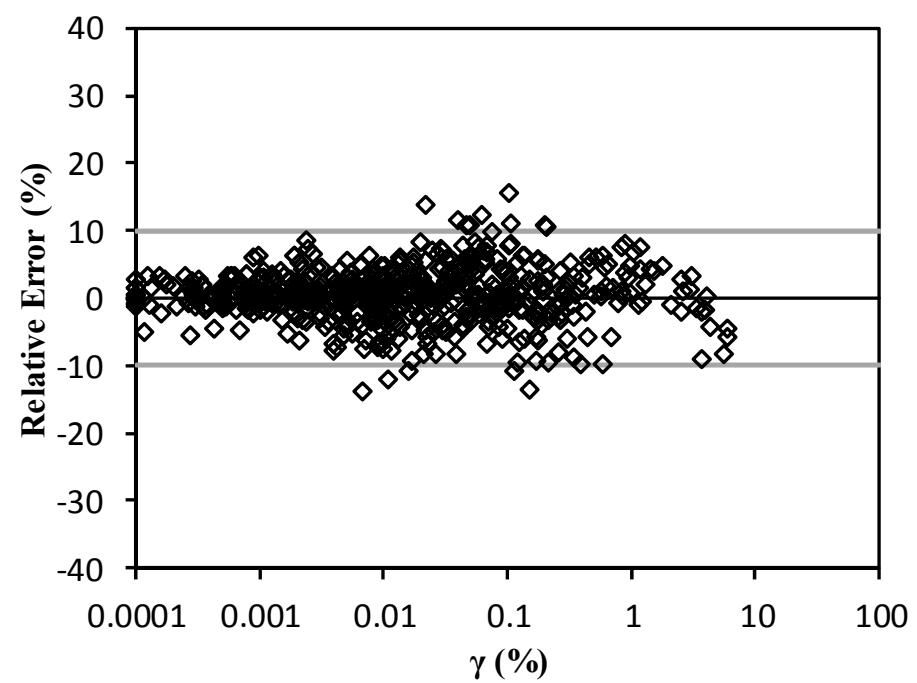

Fig. 8 Difference between the experimental and RBF-based predicted values of $\mathrm{G} / \mathrm{G}_{\max }$ with respect to $\gamma$.

Comparison between the results of the parametric study for $\gamma$ and PI (Fig. 6) and their study confirms that predictions of the proposed RBF neural network model are in agreement with the experimental results.

Figure 7 shows variations of normalized shear modulus, $\mathrm{G} / \mathrm{G}_{\max }$, versus confining pressure, $\sigma_{0}{ }_{0}$, at different levels of shear strain, $\gamma$. According to this figure, $\mathrm{G} / \mathrm{G}_{\max }$ increases with increasing $\sigma_{0}^{\prime}$ and decreasing $\gamma$. This finding is in qualitatively good agreement with the results of laboratory studies carried out by some researchers (e.g., Kagawa, 1992; Lanzo et al., 1997).

Besides, difference between the measured $\mathrm{G} / \mathrm{G}_{\max }$ and predicted values, as relative error in percentage, was plotted with respect to shear strain, $\gamma$, for all element tests data in Figure 8. It is observed that the developed model can predict the $\mathrm{G} / \mathrm{G}_{\max }$ of cohesive soils with reasonable accuracy because the residuals are satisfactorily distributed between two lines with $\pm 10 \%$ relative error.

\section{COMPARISON WITH THE PREVIOUS STUDIES}

Predictions of some previously published equation (Ishibashi and Zhang, 1993) or curves (Vucetic and Dobry, 1991; Stokoe et al., 1999) are compared with the $\mathrm{G} / \mathrm{G}_{\max }$ values predicted by the current model. Ishibashi and Zhang (1993) collected a database of experimental results on dynamic shear modulus and damping ratio. They presented a relationship for normalized shear modulus of cohesive soils in terms of cyclic shear strain, effective confining pressure, and plasticity index. Shear modulus reduction data of the currently used database (measured data) are plotted along with the predictions of RBF-based model and Ishibashi and Zhang (1993)'s relationship in Figure 9. It is clear that the relationship 


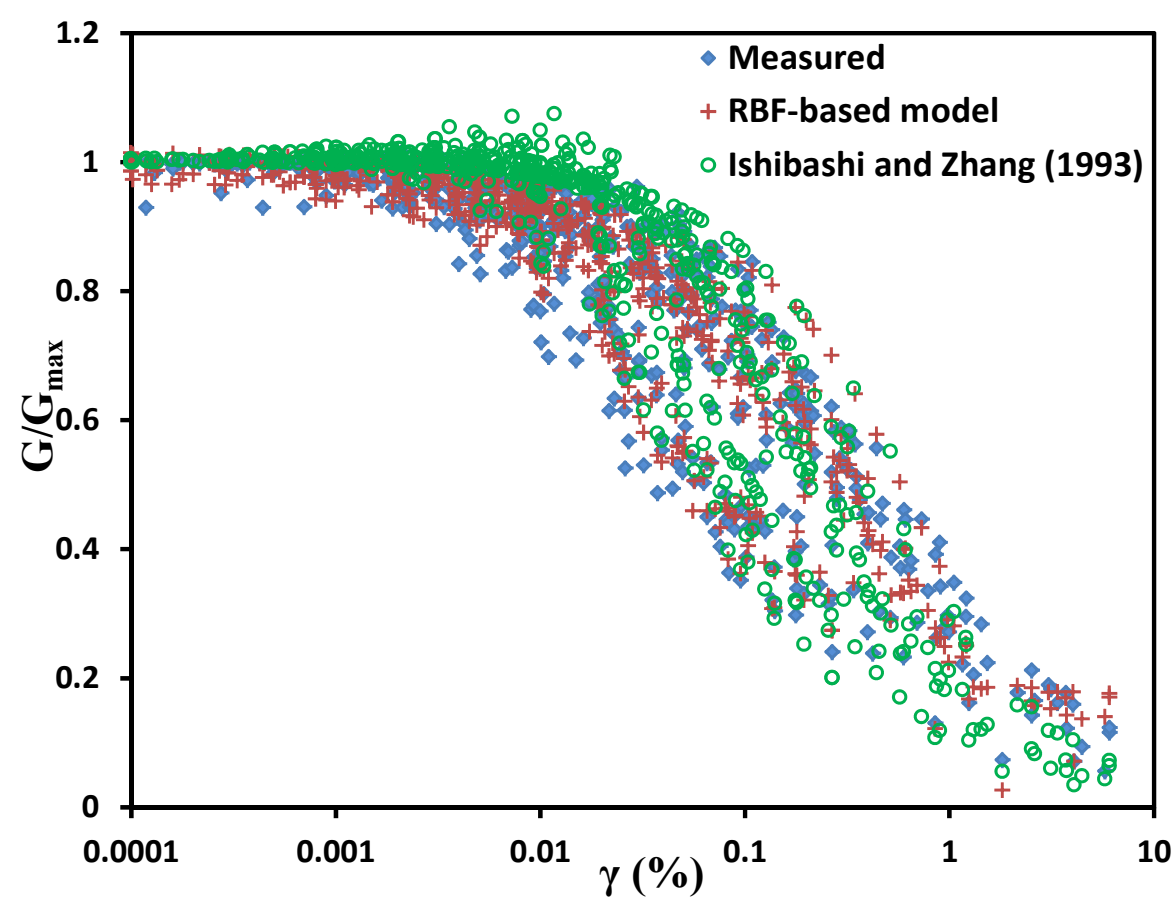

Fig. 9 Comparison of RBF-based model with Ishibashi and Zhang (1993) equation.

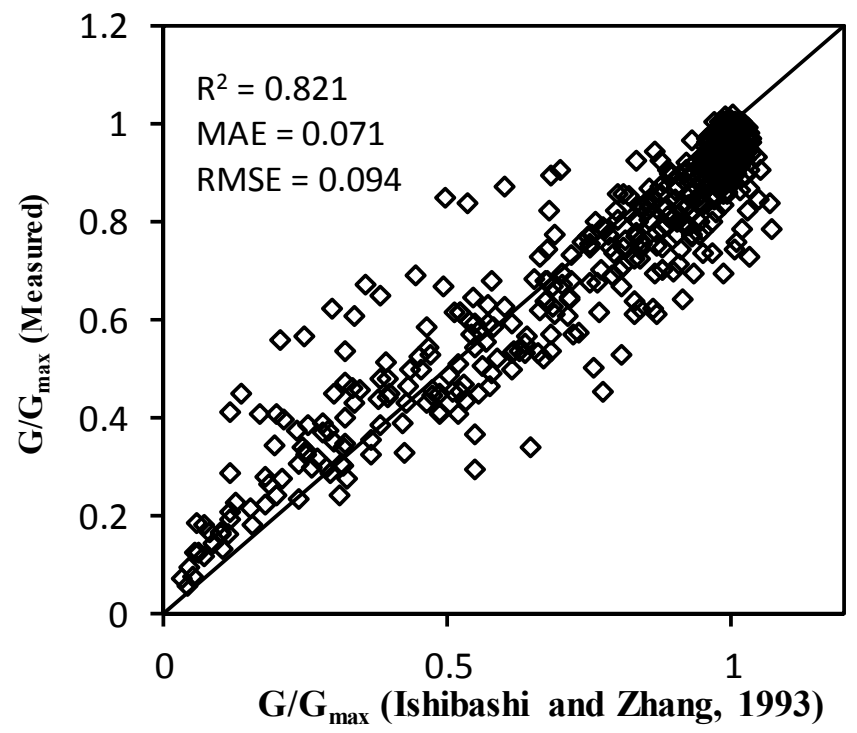

Fig. 10 Measured versus predicted values of $G / G_{\max }$ by Ishibashi and Zhang (1993) equation for all element tests data.

overestimates the values of normalized shear modulus, especially in small to moderate shear strain amplitudes. For further examination, Figure 10 was plotted to depict the measured values of $\mathrm{G} / \mathrm{G}_{\max }$ versus those predicted by Ishibashi and Zhang's equation for all element tests data. The values of $\mathrm{R}^{2}$, MAE, and RMSE were obtained equal to $0.821,0.071$, and 0.094, respectively. As shown in this figure, for $\mathrm{G} / \mathrm{G}_{\max }$ in the range of 0.8 to 1 , values of $\mathrm{G} / \mathrm{G}_{\max }$ are over-predicted, and for other values are underpredicted.
It is clearly seen in Figure 11 that accuracy of their relationship decreases with increasing shear strain and the relative error reaches about $40 \%$, whereas this value was about $10 \%$ for RBF neural network model (Fig. 8).

Table 3 presents the values of R2, RMSE, and MAE for the proposed RBF model and the $G / G_{\max }$ values estimated by Ishibashi and Zhang (1993)'s equation for cohesive soils. The results presented in this Table confirm higher precision of the proposed model with respect to the equation. It is required to 


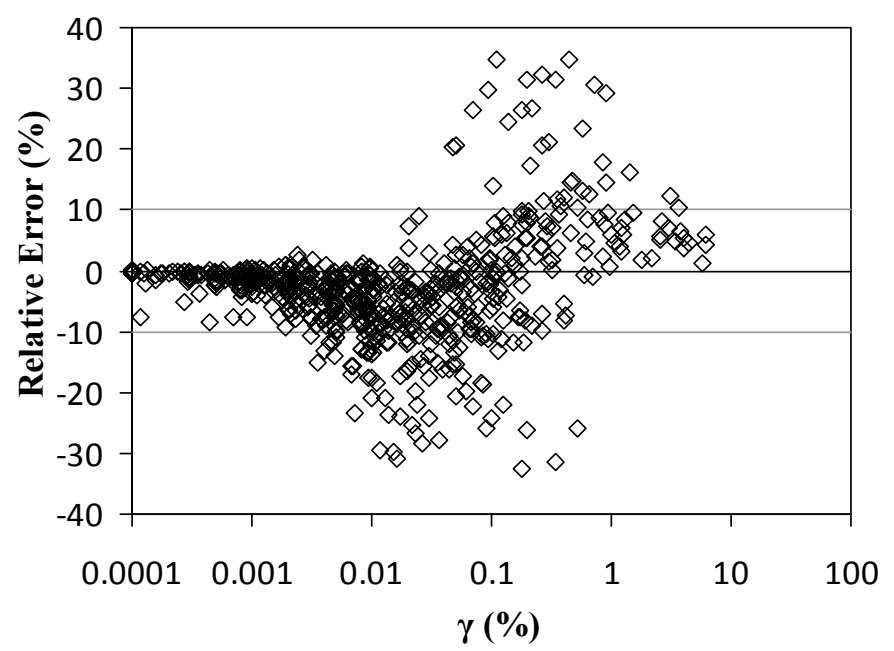

Fig. 11 Difference between the experimental and predicted values of $G / G_{\max }$ by Ishibashi and Zhang (1993) equation with respect to $\gamma$.

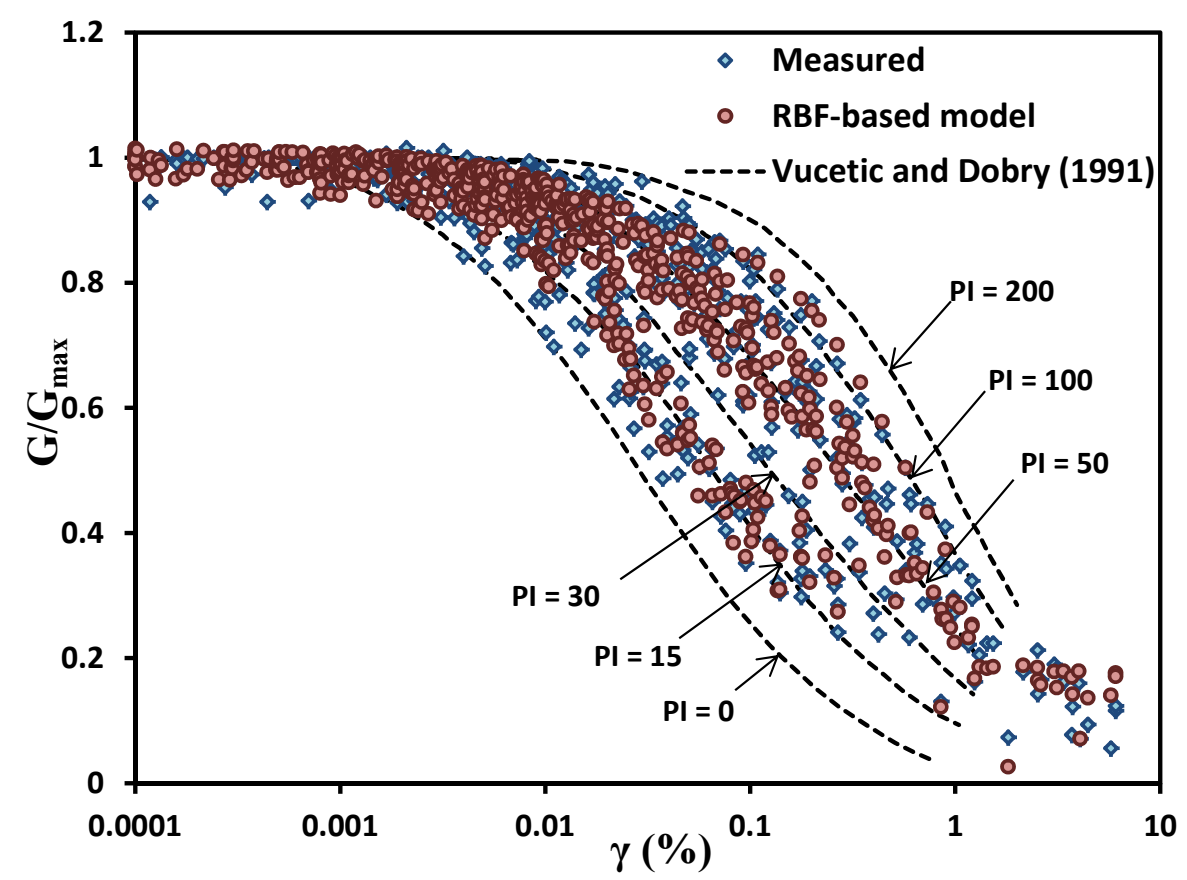

Fig. 12 Comparison of RBF-based model with curves of Vucetic and Dobry (1991).

Table 3 Comparison between values of $\mathrm{R}^{2}$, MAE, and RMSE for RBF model and previous study.

\begin{tabular}{llll}
\hline Model & \multicolumn{3}{l}{ Performance } \\
& $\mathrm{R}^{2}$ & MAE & RMSE \\
\hline $\begin{array}{l}\text { Ishibashi and Zhang } \\
\text { (1993) }\end{array}$ & 0.821 & 0.071 & 0.094 \\
$\begin{array}{l}\text { RBF-based model } \\
\text { all element tests data }\end{array}$ & 0.967 & 0.028 & 0.042 \\
$\begin{array}{l}\text { RBF-based model } \\
\text { centrifuge data }\end{array}$ & 0.923 & 0.041 & 0.044 \\
\hline
\end{tabular}

notice that the current study has employed a database larger than that used by Ishibashi and Zhang (1993). In addition, the proposed RBF model was developed by training of this larger database. Therefore, superior performance of the proposed model is reasonable and expectable.

Comparison is also made between the values predicted by RBF neural network model and the $\mathrm{G} / \mathrm{G}_{\max }-\gamma$ curves proposed by Vucetic and Dobry (1991) in Figure 12. The figure demonstrates that the predicted and the measured data points are distributed in the range of the proposed curves. 


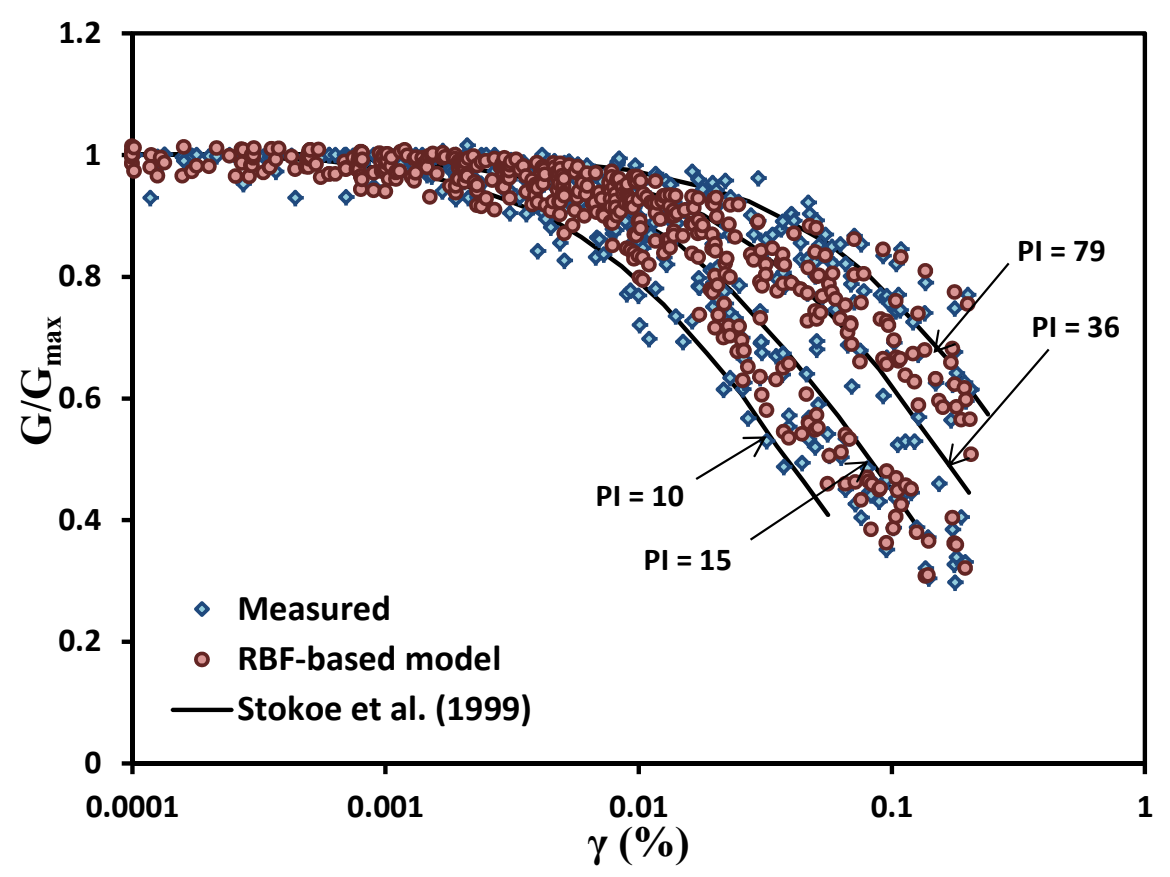

Fig. 13 Comparison of RBF-based model with curves of Stokoe et al. (1999).

Similar comparison is also made for the $\mathrm{G} / \mathrm{G}_{\max }-\gamma$ curves proposed by Stokoe et al. (1999) in Figure 13. They presented normalized shear modulus curves of soils with range of plasticity $10 \%$ to $79 \%$. Therefore, part of the database in which PI is less than $80 \%$ was selected for comparison in this figure.

Capability of the proposed ANN model is demonstrated through Figures 9, 12, and 13, in which some well-known normalized curves and equation are compared. The developed neural network model is proposed as an applicable and more reliable tool for predicting normalized shear modulus of cohesive soils because it was developed using a comprehensive database of the previously documented results.

\section{SUMMARY AND CONCLUSIONS}

Dynamic properties play a vital role in the design of structures subjected to dynamic loads. In this paper, a relatively large database was used, which contains results of cyclic tests on cohesive soils: resonant column, simple shear, cyclic torsional shear, and cyclic triaxial tests. A powerful artificial intelligence tool (i.e., radial basis function neural network) was utilized to develop a model, which obtains normalized shear modulus. Based on the experimental observations in the previous studies on cohesive soils, three parameters; shear strain, confining pressure, and plasticity index were used as input parameters to develop the RBF model. In addition, results of several centrifuge tests, not used during model development, were employed for further validation of the model. The proposed model showed a reasonably good performance for all elements and centrifuge data sets.
A parametric analysis was performed to investigate the behavior of the RBF model under different conditions and to compare model behavior with those observed in experimental studies. The results showed that $\mathrm{G} / \mathrm{G}_{\max }$ increases due to increasing $\sigma^{\prime}{ }_{0}$ and PI, while other parameters were kept constant. These trends of the developed model in the parametric analysis were in good agreement with the previously published experimental results.

A comparison between performance of the developed model and some previously published relationship and charts was done. It was clearly observed that the RBF neural network model yields a much better performance than the previous relationship and charts. The developed model facilitates estimation of soil shear modulus for the routine geotechnical projects for a wide range of cohesive soils.

\section{ACKNOWLEDGEMENT}

The authors are very grateful to Professor Ronald D. Andrus for his efficient helps in preparing database of this study.

\section{REFERENCES}

Alexander, I. and Morton, H.: 1993, Neurons and symbols: the staff that mind is made of Chapman and Hall, London.

Anderson, J.A.: 1995, An introduction to neural networks. A bradford book, MIT Press, Cambridge, MA, USA.

Arbib, M.A.: 1995, Handbook of brain theory and NN. MIT Press, MA, USA.

Baziar, M.H. and Jafarian, Y.: 2007, Assessment of liquefaction triggering using strain energy concept and 
ANN model; capacity energy. Soil Dynamics and Earthquake Engineering, 27, 1056-1072.

DOI: $10.1016 / j$.soildyn.2007.03.007

Baziar, M.H., Jafarian, Y., Shahnazari, H., Movahed, V. and Tutunchian, M.A.: 2011, Prediction of strain energybased liquefaction resistance of sand-silt mixtures: An evolutionary approach. Computers \& Geosciences, 37 , $1883-1893$.

DOI: 10.1016/j.cageo.2011.04.008

Borden, R.H., Sbal, L. and Gusta, A.: 1994, Construction related vibrations Report FHWA/NC/94-007 Washington DC.

Borden, R.H., Shao, L. and Gupta, A.: 1996, Dynamic properties of Piedmont residual soils. J Geotech Eng., 122(10), 813-821.

DOI: $10.1061 /(A S C E) 0733-9410(1996) 122: 10(813)$

Brennan, A.J., Thusyanthan, N.I. and Madabhushi, S.P.G.: 2005, Evaluation of shear modulus and damping in dynamic centrifuge tests. J Geotech Geoenviron Eng ASCE, 131(12), 1488-1497.

DOI:10.1061/(ASCE)1090-0241(2005)131:12(1488)

Broomhead, D.S. and Lowe, D.: 1988, Multivariable functional interpolation and adaptive networks. Complex Syst., 2, 321-355.

Caserta, A., Martino, S., Bozzano, F., Govoni, A. and Marra, F.: 2012, Dynamic properties of low velocity alluvial deposits influencing seismically-induced shear strains: the Grottaperfetta valley test-site (Rome, Italy). Bull Earthquake Eng. DOI: $10.1007 / \mathrm{s} 10518-012-9349-8$.

Darendeli, M.B.: 2001, Development of a new family of normalized modulus reduction and material damping curves. PhD dissertation, University of Texas at Austin, Austin, Texas.

Fausett, L.V.: 1994, Fundamentals neural networks: Architecture, algorithms, and applications. PrenticeHall, Englewood Cliffs, New Jersey.

Galushkin, A.I.: 2007, Neural Networks Theory. SpringerVerlag Berlin Heidelberg, New York.

Ghorbani, A., Jafarian, Y. and Maghsoudi, M.S.: 2012, Estimating shear wave velocity of soil deposits using polynomial neural networks: Application to liquefaction. Computers and Geosciences, 44, 86-94. DOI: $10.1016 /$ j.cageo.2012.03.002

Girosi, F, Poggio, T. and Caprile, A.: 1991, Extensions of a theory of networks for approximations and learning: outliers and negative examples. Adv Neural Inf Process Syst., 3, 750-756.

Hardin, B.O. and Drenvich, V.P.: 1972a, Shear modulus and damping in soils; design equations and curves. J Soil Mech Found Div ASCE, 98, SM7, 667-692.

Hardin, B.O. and Drnevich, V.P.: 1972b, Shear modulus and damping in soils; Measurement and parameter effects. J Soil Mech Found Div ASCE, 98, SM6, 603-624.

Hardin, B.O. and Kalinami, M.E.: 2005, Estimating the shear modulus of gravelly soils. J Geotech Geoenviron Eng ASCE, 131(7), 867-875. DOI:10.1061/(ASCE)1090-0241(2005)131:7(867)

Hartman, E.J., Keller, J.D. and Kowalski, J.M.: 1990, Layered neural networks with gaussian hidden units as universal approximations. Neural Comput, 2, 210215.

DOI:10.1162/neco.1990.2.2.210

Hornik, K., Stichcombe, M. and White, H.: 1989, Multi layers feed forward networks are universal approximation. Neural Networks, 2, 359-366. DOI: 10.1016/0893-6080(89)90020-8
Ishibashi, I. and Zhang, X.: 1993, Unified dynamic shear moduli and damping ratios of sand and clay. Soils and Foundations, 33(1), 182-191.

Jafarian, Y., Kermani, E. and Bazar, M.H.: 2010, Empirical predictive model for the $\mathrm{v}_{\max } / \mathrm{a}_{\max }$ ratio of strong ground motions using genetic programming. Computers \& Geosciences, 36(12), 1523-1531. DOI:10.1016/j.cageo.2010.07.002

Jafarian, Y., Vakili, R. and Sadeghi, A.: 2013, Prediction of cyclic resistance ratio for silty sands and its applications in the simplified liquefaction analysis. Computers and Geotechnics, 52, 54-62 DOI: 10.1016/j.compgeo.2013.04.001

Kagawa, T.: 1992, Moduli and damping factors of soft marine clay. J Geotech Eng ASCE, 118(9), 13601375.

DOI: 10.1061/(ASCE)0733-9410(1992)118:9(1360)

Kokusho, T., Yoshida, Y. and Esashi, Y.: 1982, Dynamic properties of soft clay for wide strain range. Soils and Foundations, 22(4), 1-18.

Konno, T., Hatanaka, M., Ishihara, K. and Ibe Y Iizuka, S.: 1994, Gravelly soil properties evaluation by large scale in-situ cyclic shear tests. Ground failures under seismic conditions Geotech Spec Publ ASCE, No. 4, 177-200.

Lanzo, G., Vucetic, M. and Doroudian, M.: 1997, Reduction of shear modulus at small strains in simple shear. J Geotech Geoenviron Eng ASCE, 123(11), 1035-1042. DOI:10.1061/(ASCE)1090-0241(1997)123:11(1035)

Masters, T.: 1993, Practical neural network recipes in C++. San Diego, Academic press.

McCombie, P. and Wilkinson, P.: 2002, The use of the simple genetic algorithm in finding the critical factor of safety in slope stability analysis. Computers and Geotechnics, 29, 699-714.

DOI: 10.1016/S0266-352X(02)00027-7

Menq, F.Y.: 2003, Dynamic Properties of Sandy and Gravelly Soils. PhD dissertation, University of Texas at Austin, Austin, Texas.

Penumadu, D. and Zhao, R.: 1999, Triaxial compression behavior of sand and gravel using artificial neural networks (ANN). J. Computers and Geotechnics, 24, 207-230. DOI: 10.1016/S0266-352X(99)00002-6

Richard, F.E., Hall, J.R. and Woods, R.D.: 1970, Vibrations of soils and foundations. Prentice-Hall, Englewood Cliffs NJ.

Saxena, S.K. and Reddy, K.R.. 1989, Dynamic moduli and damping ratios for monterey No.0 sand by resonant column tests. Soils and Foundations, 29(2), 37-51.

S\&ME Inc.: 1993, Seismic soil pile interaction analysesMark Clark expressway/Stono River crossings, Charleston, South Carolina. Project Rep. No. 1131-92162, Mount Pleasant SC.

S\&ME Inc. : 1998, Second report of seismic analysisDaniel Island terminal, Charleston, South Carolina. Project Rep. No. 1131-97-741, Mount Pleasant SC.

Senetakis, K., Anastasiadis, A. and Pitilakis, K.: 2012, The small-strain shear modulus and damping ratio of quartz and volcanic sands. Geotech Testing J ASTM, 35 (6). DOI: 10.1520/GTJ20120073.

Senetakis, K., Anastasiadis, A., Pitilakis, K. and Souli, A.: 2012, Dynamic behavior of Sand/Rubber mixtures, Part II: effect of rubber content on G/G0- $\gamma$-DT curves and volumetric threshold strain. J ASTM Int., 9(2). DOI: 10.1520/JAI103711.

Shahin, M.A., Maier, H.R. and Jaksa, M.B.: 2002, Predicting settlement of shallow foundations using 
neural networks. J Geotech Geoenviron Eng ASCE, 128 (9), 785-93.

DOI:10.1061/(ASCE)1090-0241(2002)128:9(785)

Smith, M.: 1993, Neural networks for statistical modeling. Van Nostrand Reinhold, New York.

Stokoe, K.H.II., Darendeli, M.B., Gilbert, R.B., Menq, F.Y. and Choi, W.K.: 2004, Development of a new family of normalized modulus reduction and material damping curves. Proc. NSF/PEER Int. Workshop on Uncertainties in Nonlinear Soil Properties and their Impact on Modeling Dynamic Soil Response, University of California at Berkeley, Berkeley, California.

Stokoe, K.H.II., Hwang, S.K., Darendeli, M.B. and Lee, N.J.: 1995, Correlation study of nonlinear dynamic soils properties. Final Rep. Westinghouse Savannah River Company, Aiken, SC.

Stokoe, K.H.II., Hwang, S.K., Lee, N.J. and Andrus, R.D.: 1994, Effects of various parameters on the stiffness and damping of soils at small to medium strains. Proc. Int. Symp. Prefailure Deformation Characteristics of Geomaterials, Sapporo, Japan, 2, 785-816.

Stokoe, K.H.II., Darendeli, M.B., Andrus, R.D. and Brown, L.T.: 1999, Dynamic soil properties: Laboratory, field and correlation studies. Proc. 2nd Int. Conf. on Earthquake Geotechnical Engineering, Lisbon, Portugal, 3, 811-845.

Tatsuoka, F., Iwasaki, T., Fukushima, Sh. and Sudo, H.: 1979, Stress conditions and stress histories affecting shear modulus and damping of sand under cyclic loading. Soils and Foundations, 19(2), 29-43.

U.S. Army Corps of Engineers, USACE: 1978, Dynamic test report sheets on soil samples for Richard B. Russell Dam. Rep. No. EN-FS-77-84 South Atlantic Division Laboratory Marietta Ga.

U.S. Army Corps of Engineers, USACE: 1979, Design Memorandum 17, Richard B. Russell Dam and Lake, Savannah River, Georgia and South Carolina. Earth embankments Vol. IV, Appendix VIII, Savannah District, Savannah Ga.
Vucetic, M. and Dobry, R.: 1991, Effect of soil plasticity on cyclic response. J Geotech Eng ASCE, 117(1), 89107. DOI: 10.1061/(ASCE)0733-9410(1991)117:1(89)

Wasserman, P.D.: 1993, Advanced methods in neural computing. New York: Prentice Hall Company, Van Nostrand Reinhold.

Wilson, J.M.R.: 1988, A theoretical and experimental investigation into the dynamic behavior of soils. $\mathrm{PhD}$ thesis Uni. of Cambridge, Cambridge, U.K.

Xenaki, V.C. and Athanasopoulos, G.A.: 2008, Dynamic properties and liquefaction resistance of two soil materials in an earthfill dam-laboratory test results. Soil Dynamic and Earthquake Engineering, 28, 605620. DOI: 10.1016/j.soildyn.2007.10.001

Yasuda, N. and Matsumoto, N.: 1993, Dynamic deformation characteristics of sand and rockfill materials. Can Geotech J., 30, 747-757.

Yoshimi, Y., Tokimatsu, K., Kaneko, O. and Makihara, Y.: 1984, Undrained cyclic strength of a dense Niigata sand. Soils and Foundations, 24(4), 131-145.

Zhang, J., Andrus, R.D. and Juang, CH.: 2005, Normalized shear modulus and material damping ratio relationships. J. Geotech Geoenviron Eng ASCE, 131(4), 453-464. DOI:10.1061/(ASCE)1090-0241(2005)131:4(453) 